\title{
Suppression of Apolipoprotein B Secretion from HepG2 Cells by Glucosyl Hesperidin
}

\author{
Yoshikatsu MiwA ${ }^{1}$, Hitoshi MitsuZumi ${ }^{1, *}$, Mika YAMAdA $^{1}$, Norie ARAI ${ }^{1}$, Fujimi TANABE ${ }^{1}$, \\ Katsuhide OKADA ${ }^{2}$, Michio KUBOTA ${ }^{1}$, Hiroto CHAEN $^{1}$, Takahiro SunAYAMA ${ }^{3}$ \\ and Masayoshi KIBATA ${ }^{1,4}$ \\ ${ }^{1}$ Hayashibara Biochemical Laboratories, Inc., 1-2-3 Shimoishii, Okayama 700-0907, Japan \\ ${ }^{2}$ Hayashibara Co., Ltd., 7-7 Amase Minami-machi, Okayama 700-0834, Japan \\ ${ }^{3}$ Dai-ichi Central Bldg. Clinic, 6-36-601 Honmachi, Okayama 700-0901, Japan \\ ${ }^{4}$ National Hospital Organization Minami-Okayama Medical Center, 4066 \\ Hayashima, Okayama 701-0304, Japan
}

(Received March 27, 2006)

\begin{abstract}
Summary Our previous study has shown that a soluble hesperidin derivative, glucosyl hesperidin (G-hesperidin), preferentially lowers serum triglyceride (TG) level in hypertriglyceridemic subjects through the improvement of very low-density lipoprotein (VLDL) metabolic abnormality. G-Hesperidin has also been found to decrease an elevated serum apolipoprotein B (apo B) level in the hypertriglyceridemic subjects, suggesting a possibility that this compound suppresses excess VLDL secretion in the liver. In the present study, to gain a better understanding of possible mechanisms by which G-hesperidin lowers serum TG, we examined whether this derivative affects apo B secretion from HepG2 human hepatoma cells, a model of hepatic VLDL secretion. As a result, G-hesperidin significantly reduced apo B secretion from the oleate-stimulated HepG2 cells. Furthermore, G-hesperidin significantly suppressed apo B secretion only in the oleate-stimulated cells and failed to act on the cells incubated without oleate. In the oleate-stimulated cells, G-hesperidin significantly decreased cellular cholesteryl ester (CE), although it had no effect on cellular TG or free cholesterol amounts. Moreover, the oleate-stimulated cells had a decrease in cellular apo B amounts by G-hesperidin exposure. These findings indicate that G-hesperidin downregulates the assembly of apo B-containing lipoproteins via the reduction of CE synthesis augmented with oleate and results in suppressing excess apo B secretion from the cells. This effect is speculated to be associated with the improvement of VLDL metabolic abnormality in hypertriglyceridemic subjects and considered as a mechanism of lowering serum TG.
\end{abstract}

Key Words glucosyl hesperidin, very low-density lipoprotein, apolipoprotein B, cholesteryl ester, HepG2 cells

Hesperidin, a citrus flavonoid, is well known to decrease capillary fragility and permeability (1). This compound has also been reported to have various physiological activities including antioxidant effect (1), antihypertensive effect (2) and antiallergic effect (3). Therefore, hesperidin has attracted attention as a multifunctional food ingredient. However, the use of this flavonoid in the field of foods has been limited because of its low water solubility.

To solve this problem, Hijiya and Miyake have synthesized a soluble derivative of hesperidin, glucosyl hesperidin (G-hesperidin), by regioselective transglycosylation with cyclodextrin glucanotransferase from Bacillus stearothermophilus (Fig. 1) (4). G-Hesperidin is very soluble in water, and its solubility is about 10,000 times greater than that of conventional hesperidin (4). Moreover, it has been verified that this derivative releases hesperidin through hydrolysis by brush border $\alpha$-glu-

\footnotetext{
* To whom correspondence should be addressed.

E-mail: hmitsu@hayashibara.co.jp
}

cosidases $(4,5)$. Our recent studies have shown that Ghesperidin as well as hesperidin exhibits such physiological activities as decrease of serum lipid peroxide level in hyperlipidemic mice (6), antihypertensive effect on spontaneously hypertensive rats $(7,8)$ and suppression of passive cutaneous anaphylaxis in rats (9). These findings indicate that G-hesperidin behaves as hesperidin in the body and results in the exhibition of physiological activities. Thus, G-hesperidin is considered to be available effectively as a hesperidin source.

Furthermore, we have found that the supplementation of G-hesperidin markedly lowered serum triglyceride (TG) in mice with hyperlipidemia induced by a highfat diet (6). Such TG-lowering effect has also been observed in humans; it has been confirmed that G-hesperidin preferentially lowered serum TG level in hypertriglyceridemic subjects $(10,11)$. Hypertriglyceridemia has been known to be attributed to the accumulation of serum TG-rich lipoproteins such as very low-density lipoprotein (VLDL) and its metabolites, remnant lipoproteins (12-14). The accumulation of these TG-rich 


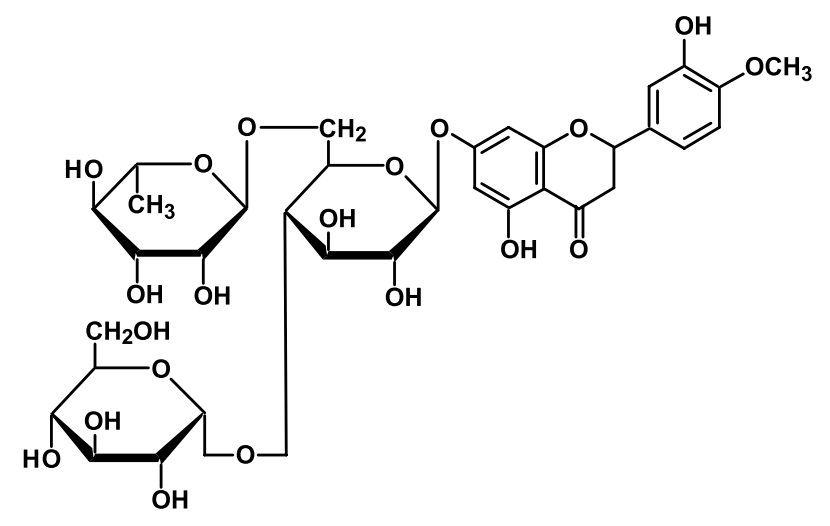

Fig. 1. Chemical structure of glucosyl hesperidin (Ghesperidin).

lipoproteins in serum is considered to be caused by an imbalance of the synthesis and catabolism of VLDL, namely, VLDL metabolic abnormality (12-14). In the previous clinical study, we have found that G-hesperidin administration reduced serum VLDL and remnant lipoproteins in the hypertriglyceridemic subjects (11). From this result, it is suggested that the TG-lowering effect of G-hesperidin is due to the improvement of VLDL metabolic abnormality.

In addition, G-hesperidin administration significantly decreased an elevated serum apolipoprotein B (apo B) level in hypertriglyceridemic subjects (11). It has been demonstrated that apo B forms VLDL by complexing with TG and cholesteryl ester (CE) in hepatocytes and is secreted from the liver (15-18). On the basis of this finding, serum apo B level is regarded as an index of hepatic VLDL secretion. Thus, our observation implies a possibility that G-hesperidin suppresses excess VLDL secretion in the liver.

The present study was undertaken to clarify the mechanisms of the TG-lowering effect of G-hesperidin. In particular, we investigated the effect of G-hesperidin on hepatic VLDL secretion to assess the possibility mentioned above. For experiments, the human hepatoma cell line, HepG2, was used as a model of VLDL secretion from the liver. HepG2 cells have been recognized as an important in vitro model system to understand the regulatory processes involved in the assembly of apo B and lipids in hepatic VLDL synthesis and secretion. In these cells, it has been demonstrated that apo B forms relatively smaller lipoprotein particles by complexing with lipids and is secreted into the culture medium (19-21). Nevertheless, such apo B-containing lipoprotein particles were recently shown to be produced through the lipolysis of secreted VLDL by HepG2 cell-derived lipase $(22,23)$. Hence, it is considered that apo B measurement in the culture medium of HepG2 cells is a reflection of VLDL secretion $(22,23)$. Using this hepatocyte model system, we examined whether G-hesperidin affects apo B secretion.

\section{MATERIALS AND METHODS}

1. Materials. HepG2 cells (ATCC HB-8065) were obtained from the American Type Culture Collection (Rockville, MD, USA). Dulbecco's modified Eagle's medium (DMEM) and Dulbecco's phosphate-buffered saline (D-PBS, $\mathrm{Ca}^{2+}$ and $\mathrm{Mg}^{2+}$ free) were purchased from Nissui Pharmaceutical Co. Ltd. (Tokyo, Japan). Fetal bovine serum (FBS) was obtained from Moregate Biotech (Bulimba, Australia). G-Hesperidin was provided by Hayashibara Co. Ltd. (Okayama, Japan). Hesperidin and $o$-phenylenediamine were purchased from Wako Pure Chemical Industries, Ltd. (Osaka, Japan). Hesperetin, sodium oleate and bovine serum albumin (BSA, fatty acid free) were purchased from Sigma-Aldrich Corp. (St. Louis, MO, USA). Human serum albumin (HSA) was purchased from Bayer Corp. (Pittsburgh, PA, USA). Monoclonal human apo B antibody was obtained from Chemicon International Inc. (Temecula, CA, USA). Affinity purified polyclonal apo B antibody conjugated to peroxidase was from The Binding Site Ltd. (Birmingham, UK). Purified human apo B was from Calbiochem-Novabiochem International Inc. (La Jolla, CA, USA). All other regents were of analytical grade.

2. Cell culture. HepG2 cells were maintained in $25 \mathrm{~cm}^{2}$ flasks (Corning Costar Corp., Cambridge, MA, USA) in DMEM containing 10\% FBS and 100 IU penicillin-streptomycin (complete medium) at $37^{\circ} \mathrm{C}$ under $5 \% \mathrm{CO}_{2}$. For experiments, cells $\left(5 \times 10^{3}\right.$ cells $\left./ \mathrm{cm}^{2}\right)$ were plated in 24- or 6-well culture plates (Falcon Scientific, VWR, Missisauga, ON, Canada) and grown in the complete medium. After $4 \mathrm{~d}$ of growth, when the cells reached at least 30\% confluence, experiments were initiated.

3. Treatments of cells. At the beginning of each experiment, the medium was removed from culture plates, and the cells were preincubated for $24 \mathrm{~h}$ in the complete medium with the indicated concentrations of G-hesperidin or its related compounds (hesperidin and hesperetin). Then, the cells were washed twice with DPBS and incubated for a further $24 \mathrm{~h}$ in serum-free DMEM containing the same concentrations of the above compounds, $1 \%$ BSA and $0.2 \mathrm{~mm}$ oleate (oleate-BSA complex, molar ratio $1.3: 1$ ). The oleate-BSA complex was prepared according to the method of Dixon et al. (24). Hesperidin and hesperetin were dissolved in dimethyl sulfoxide (DMSO). The final concentration of DMSO in the media did not exceed $0.1 \%$, and all experimental media were adjusted for the same DMSO concentration. In some experiments, the timing of G-hesperidin addition was altered. Briefly, the cells were exposed to Ghesperidin during the indicated periods. In another experiment, the cells were incubated with or without Ghesperidin in serum-free DMEM containing 1\% BSA instead of oleate-BSA complex.

After completion of each treatment described above, the culture media were collected and immediately stored at $-20^{\circ} \mathrm{C}$ for measurement of apo B concentrations. The cells were washed twice with ice-cold D-PBS, solubilized by addition of ice-cold D-PBS containing $0.25 \%$ Triton X-100, and placed on ice for $1 \mathrm{~h}$. The cell lysates thus obtained were stored at $-20^{\circ} \mathrm{C}$ for cellular apo B, lipid and protein determinations. 
4. Apo B determination. Apo B concentrations in the culture media were measured by a sandwich enzymelinked immunosorbent assay (ELISA) according to the method of Wilcox et al. (17). In the ELISA, monoclonal human apo B antibody was used as the capture antibody, and an affinity purified polyclonal apo B antibody conjugated to peroxidase was used for detection. $o$-Phenylenediamine was used as substrate for color development, and the absorbance at $490 \mathrm{~nm}$ was determined using a microplate reader. A purified human apo B was used for generating the standard curve. The amount of secreted apo B was expressed per mg of total cellular protein.

Cellular apo B concentrations were determined as above in aliquots of cell lysates. Cell lysate samples were diluted with D-PBS to give a final Triton X-100 concentration of no greater than $0.025 \%$. This surfactant concentration did not interfere with the ELISA. The amount of cellular apo B was expressed per mg of total cellular protein.

5. Determination of cellular lipids. Cellular lipids were extracted from the cell lysates by the method of Bligh and Dyer (25). The extracted lipids were evaporated to dryness under nitrogen, redissolved in isopropanol containing 10\% Triton X-100, and used for the measurement of cellular TG, free cholesterol (FC) and total cholesterol (TC). These cellular lipids were determined by a modification of the method of Carr et al. (26) using enzymatic assay kits from Wako Pure Chemical Industries, Ltd. CE amount was calculated as the difference between TC and FC amounts. The results were expressed as cellular lipid (TG, FC or CE) amount per mg of total cellular protein.

6. Determination of cellular protein. Cellular protein was measured by the method of Bradford (27) using the Protein Assay Dye Reagent from Bio-Rad Laboratories Inc. (Hercules, CA, USA). HSA was used as a standard. Cell lysate samples were diluted with saline so that the final concentration of Triton X-100 in the samples was not greater than $0.005 \%$. This surfactant concentration did not interfere with the assay.

7. Statistical analyses. Data were expressed as means \pm SD, which were compared by one-way ANOVA. Differences among groups were determined using the Tukey-Kramer test for all experiments, with the exception of G-hesperidin requirement analysis (Fig. 5), which was examined using the Dunnett test. The significance level was set at $p<0.05$ for all tests. Statistical analyses were performed with Stat View version 5.0 for Windows (SAS Institute, Cary, NC).

\section{RESULTS}

\section{Effect of G-hesperidin on apo B secretion}

HepG2 cells were incubated with various concentrations of G-hesperidin in the presence of oleate, and the accumulation of apo B in the culture media was measured. The result is shown in Fig. 2. G-Hesperidin significantly and dose-dependently reduced the secretion of apo B into the culture media. At the concentrations of G-hesperidin used in this experiment, there were no sig-

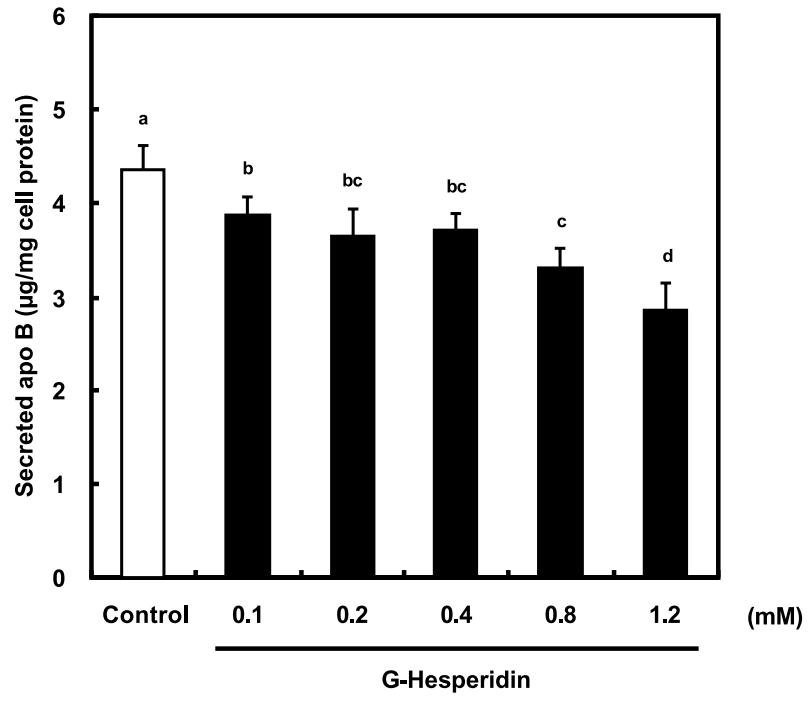

Fig. 2. Effect of G-hesperidin on apo B secretion from HepG2 cells. HepG2 cells were preincubated for $24 \mathrm{~h}$ in the complete medium with the indicated concentrations of G-hesperidin. Subsequently, the cells were incubated for a further $24 \mathrm{~h}$ in serum-free DMEM containing the same concentrations of G-hesperidin, 1\% BSA and $0.2 \mathrm{~mm}$ oleate. After completion of the incubation, apo B amounts in the culture media were measured by ELISA. Data not sharing a common letter are significantly different at $p<0.05$.
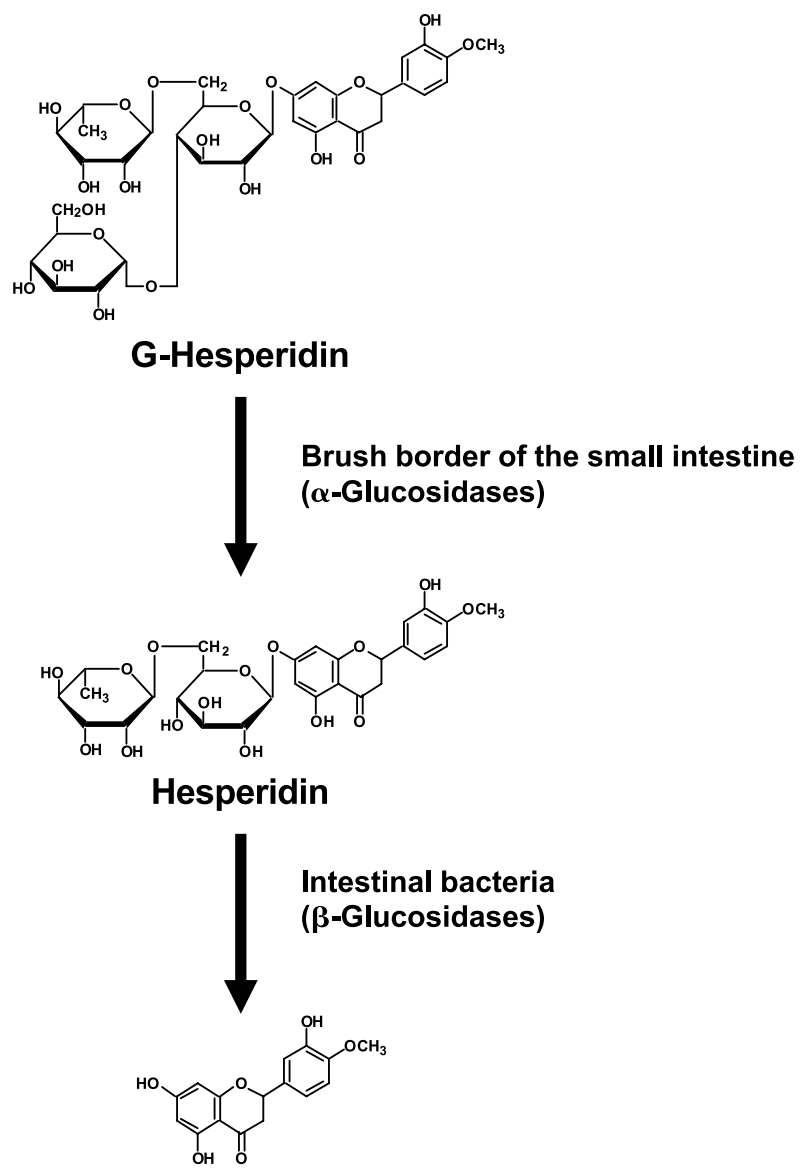

Hesperetin

Fig. 3. Possible metabolic fate of G-hesperidin in the body. 
nificant effects on total cellular protein or the number of viable cells.

2. Effects of hesperidin and hesperetin on apo B secretion G-Hesperidin has been demonstrated to release hesperidin through hydrolysis by brush border $\alpha$-glucosidases (Fig. 3) (4, 5). In addition, hesperidin has been shown to be absorbed as hesperetin after deglycosylation in the intestinal tract, thereby exhibiting physiological activities (Fig. 3) (1, 28-30). Therefore, to clarify whether the reduction of apo B secretion is caused by Ghesperidin itself or hesperidin/hesperetin released, the effects of these compounds were compared. The result is shown in Fig. 4. Hesperidin and hesperetin as well as Ghesperidin significantly suppressed apo B secretion from HepG2 cells incubated in the presence of oleate. In particular, the effect of hesperetin was more pronounced than that of G-hesperidin or hesperidin, and a distinct suppression of apo $\mathrm{B}$ secretion was observed at

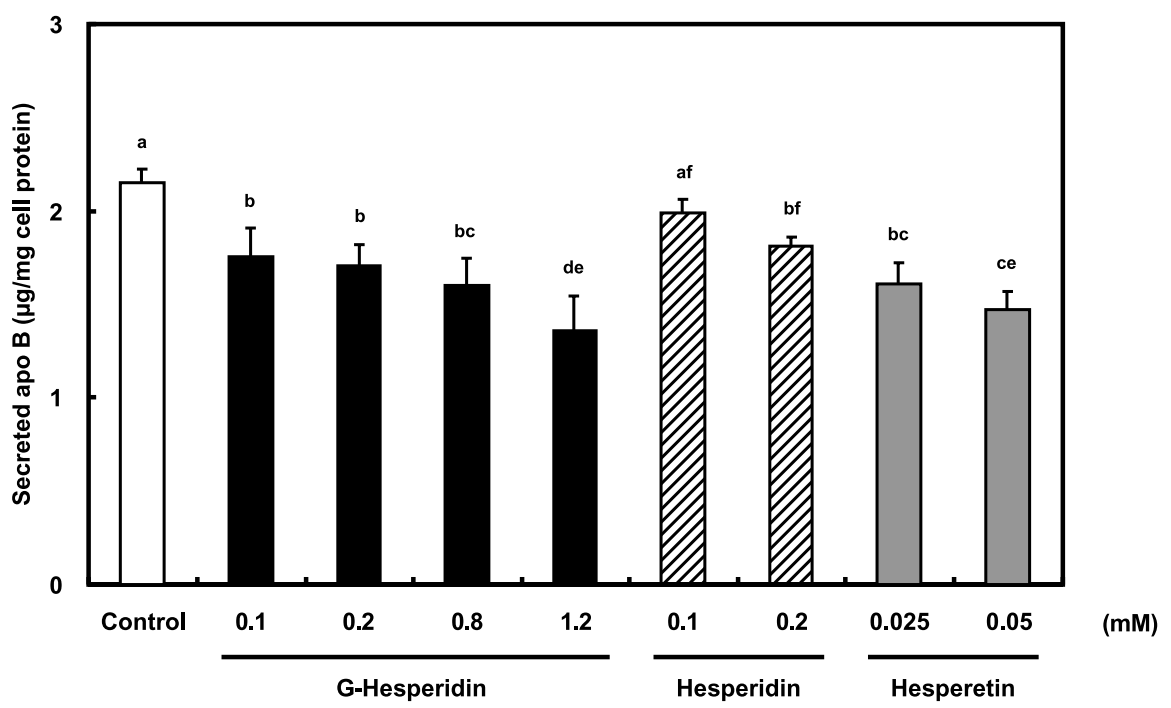

Fig. 4. Effects of G-hesperidin and its related compounds on apo B secretion from HepG2 cells. HepG2 cells were preincubated for $24 \mathrm{~h}$ in the complete medium with the indicated concentrations of G-hesperidin or its related compounds (hesperidin and hesperetin). Subsequently, the cells were incubated for a further $24 \mathrm{~h}$ in serum-free DMEM containing the same concentrations of the above compounds, $1 \%$ BSA and $0.2 \mathrm{mM}$ oleate. After completion of the incubation, apo B amounts in the culture media were measured by ELISA. Data not sharing a common letter are significantly different at $p<0.05$.

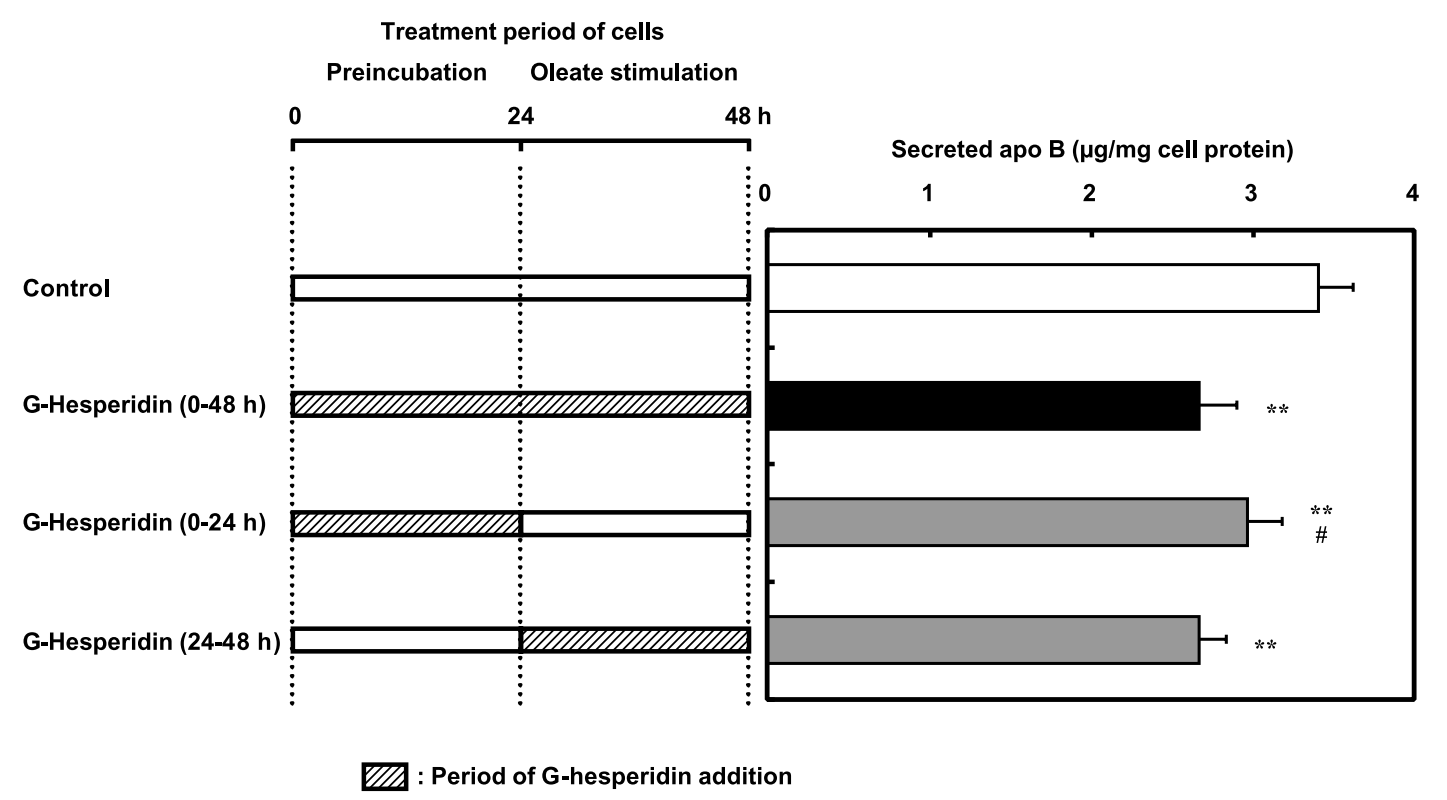

Fig. 5. Effect of G-hesperidin added to the media at different periods of the incubation on apo B secretion from HepG2 cells. HepG2 cells were preincubated for $24 \mathrm{~h}$ in the complete medium. Subsequently, the cells were incubated for a further $24 \mathrm{~h}$ in serum-free DMEM containing 1\% BSA and $0.2 \mathrm{mM}$ oleate. Time schedule for G-hesperidin addition is shown in the left side of the figure. G-Hesperidin (1.2 mM) was added to the media during the indicated periods. After completion of the incubation, apo B amounts in the culture media were measured by ELISA. ${ }^{* *} p<0.01$, as compared with the value of control. ${ }^{\#} p<0.05$, as compared with the value of G-hesperidin $(0-48 \mathrm{~h})$. 
$0.025 \mathrm{~mm}$ hesperetin. Total cellular protein and the number of viable cells were not affected by these treatments except that they were reduced by $10 \%$ at $0.05 \mathrm{~mm}$ hesperetin.

3. The time of G-hesperidin requirement for the suppression of apo $B$ secretion

To clarify the time of G-hesperidin requirement for the suppression of apo B secretion, we investigated the effect of G-hesperidin added to the media at different periods of the incubation. The result is shown in Fig. 5. When G-hesperidin was added to the media during the preincubation period and removed from the media at the subsequent oleate stimulation period, the suppressive effect on apo B secretion was small compared to that obtained by a continuous exposure of G-hesperidin. On the other hand, when G-hesperidin was absent in the media during the preincubation period and supplied to the media only during the oleate stimulation period, the suppressive effect on apo B secretion was comparable to that caused by a continuous exposure of G-hesperidin. These results suggest that the existence of G-hesperidin during the oleate stimulation period is critical for the suppression of apo B secretion.

4. G-Hesperidin suppresses apo B secretion elevated by oleate stimulation

As shown in Fig. 5, it is considered that G-hesperidin must be supplied to the cells during the oleate stimulation period for the exhibition of sufficient effect. This suggests that G-hesperidin exhibits its action in the processes of cell responses elevated by oleate stimulation. Therefore, we compared the effects of G-hesperidin on apo B secretion from HepG2 cells incubated in the presence and absence of oleate. As a result, G-hesperidin significantly suppressed apo B secretion only in the oleate-stimulated cells and failed to exhibit its effect in the absence of oleate (Fig. 6).

5. Effect of G-hesperidin on cellular lipids

It has been known that oleate augments syntheses of lipids such as TG and CE in HepG2 cells (17, 31, 32). The augmentation of these lipid syntheses facilitates the assembly of apo B-containing lipoproteins and results in increasing apo B secretion from the cells $(17,31,32)$. In contrast, the reduction of TG or CE synthesis prevents the assembly of apo B-containing lipoproteins and results in decreasing apo B secretion from the cells (17, 31,32 ). Thus, apo B secretion from HepG2 cells has been considered to be regulated by the cellular lipid syntheses $(17,31,32)$. On the basis of these findings, we examined whether G-hesperidin affects cellular lipid

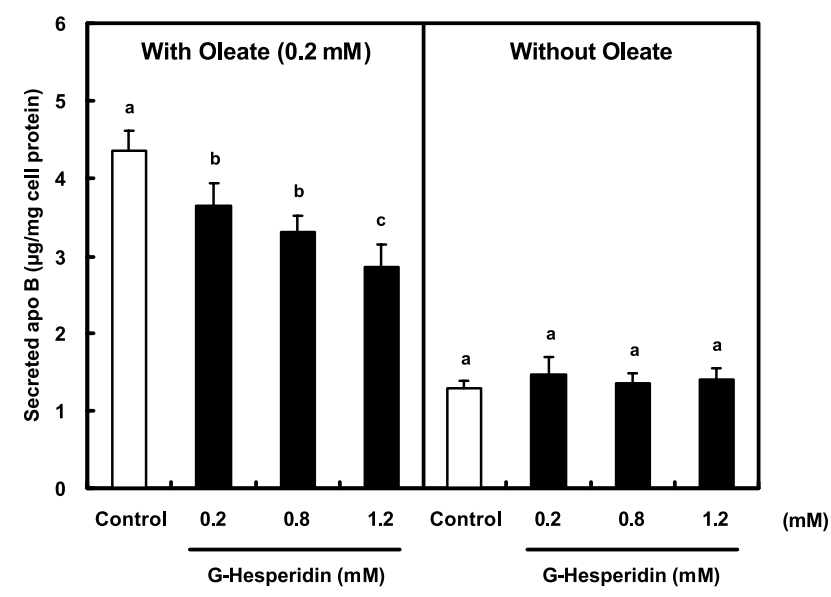

Fig. 6. Effects of G-hesperidin on apo B secretion from HepG2 cells incubated in the presence and absence of oleate. HepG2 cells were preincubated for $24 \mathrm{~h}$ in the complete medium with the indicated concentrations of G-hesperidin. Subsequently, the cells were incubated for a further $24 \mathrm{~h}$ in serum-free DMEM containing the same concentrations of G-hesperidin and 1\% BSA with or without $0.2 \mathrm{~mm}$ oleate. After completion of the incubation, apo B amounts in the culture media were measured by ELISA. Data not sharing a common letter in the same panel are significantly different at $p<0.05$.
TG

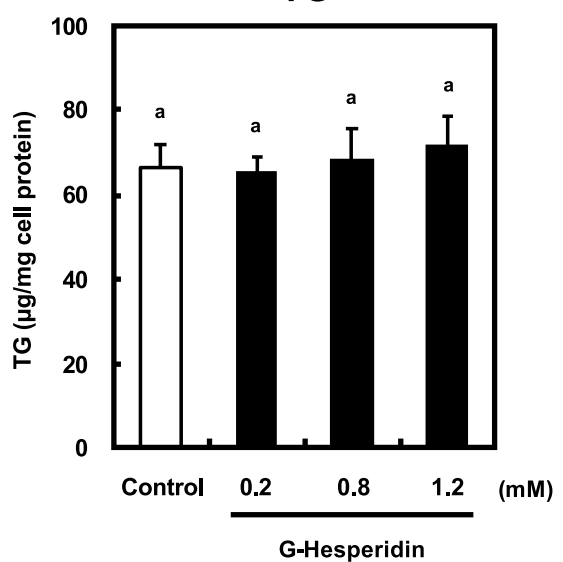

FC

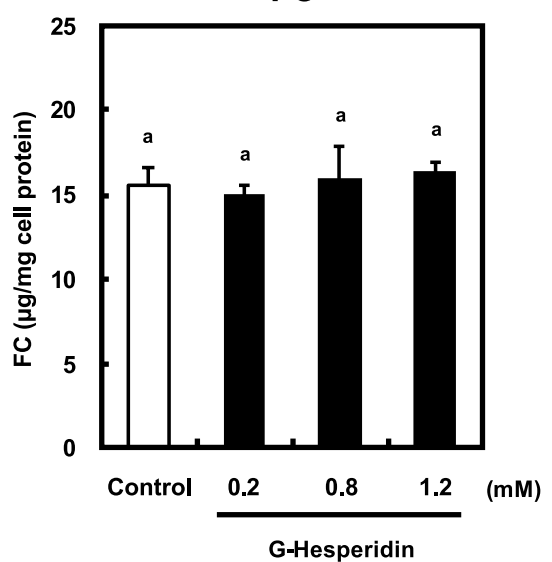

CE

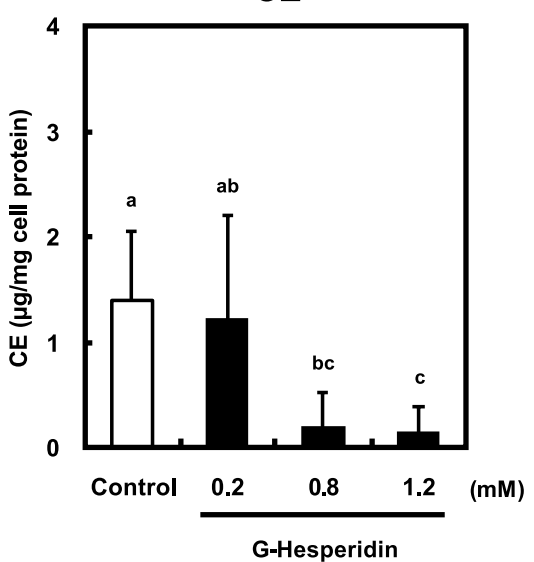

Fig. 7. Effect of G-hesperidin on cellular lipid amounts in HepG2 cells incubated in the presence of oleate. HepG2 cells were preincubated for $24 \mathrm{~h}$ in the complete medium with the indicated concentrations of G-hesperidin. Subsequently, the cells were incubated for a further $24 \mathrm{~h}$ in serum-free DMEM containing the same concentrations of G-hesperidin, $1 \%$ BSA and $0.2 \mathrm{~mm}$ oleate. After completion of the incubation, cellular lipids were extracted and quantitated by enzymatic assays. CE amount was calculated as the difference between TC and FC amounts. Data not sharing a common letter in the same panel are significantly different at $p<0.05$. 


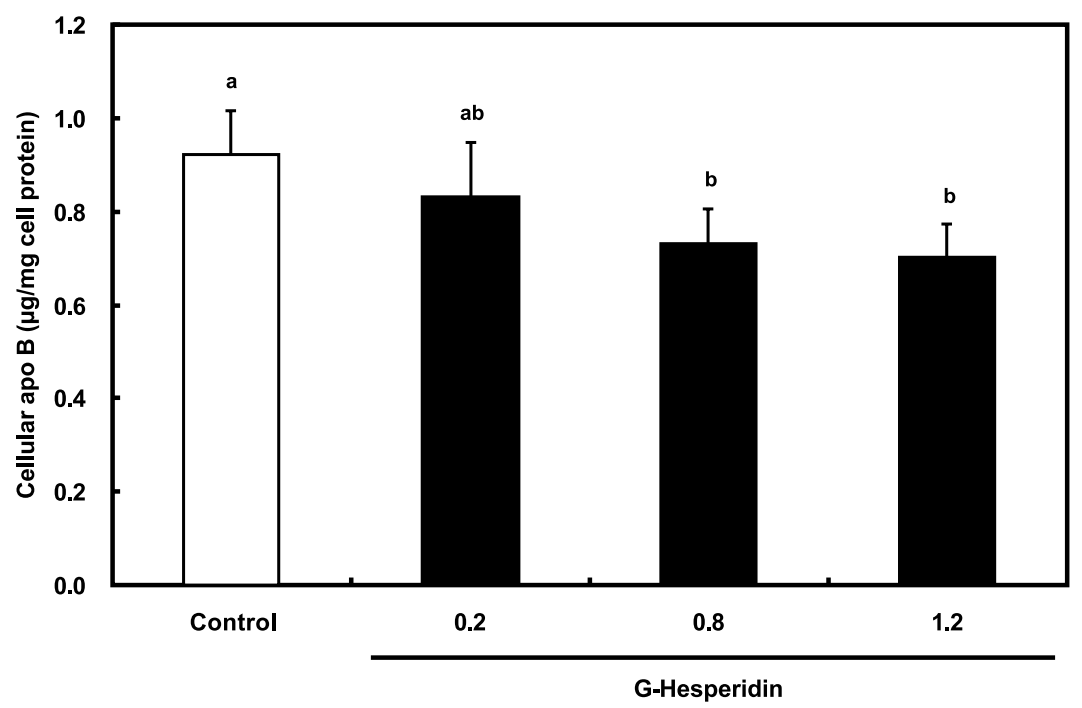

Fig. 8. Effect of G-hesperidin on cellular apo B amounts in HepG2 cells incubated in the presence of oleate. HepG2 cells were preincubated for $24 \mathrm{~h}$ in the complete medium with the indicated concentrations of G-hesperidin. Subsequently, the cells were incubated for a further $24 \mathrm{~h}$ in serum-free DMEM containing the same concentrations of G-hesperidin, $1 \%$ BSA and $0.2 \mathrm{~mm}$ oleate. After completion of the incubation, the cells were lysed, and apo B amounts in the cell lysates were measured by ELISA. Data not sharing a common letter are significantly different at $p<0.05$.

amounts in the oleate-stimulated HepG2 cells. The result is shown in Fig. 7. G-Hesperidin significantly reduced cellular CE amount, although it had no effect on cellular TG or FC amounts.

6. Effect of G-hesperidin on cellular apo B amounts

It has been verified that the reduction of cellular lipid syntheses prevents the assembly of apo B-containing lipoproteins and subsequently leads to a rapid intracellular degradation of apo B (22, 33-35). Hence, the influence of G-hesperidin on cellular apo B amounts in oleate-stimulated HepG2 cells was examined. The result is shown in Fig. 8. The cellular apo B amounts were significantly decreased in the cells exposed to G-hesperidin, and the effect had a tendency to depend on the dose level of G-hesperidin.

\section{DISCUSSION}

Our previous study has shown that G-hesperidin administration preferentially lowers serum TG level in hypertriglyceridemic subjects through the improvement of VLDL metabolic abnormality (11). G-Hesperidin has also been found to decrease a high value of serum apo B in hypertriglyceridemic subjects (11), suggesting a possibility that this compound suppresses VLDL secretion elevated in the liver.

In the present study, to gain a better understanding of possible mechanisms by which G-hesperidin lowers serum TG level, we focused on VLDL secretion in the liver and examined whether this flavonoid derivative affects apo B secretion in a HepG2 cell model system, a model of hepatic VLDL secretion. As a consequence, Ghesperidin significantly reduced apo B secretion from HepG2 cells stimulated with oleate (Fig. 2). On the basis of this observation, it is considered that G-hesperidin suppresses apo B-containing lipoprotein secretion augmented by oleate stimulation. This result agrees well with that obtained by the G-hesperidin administration test in the hypertriglyceridemic subjects mentioned above (11) and strongly supports a hypothesis that Ghesperidin suppresses excess VLDL secretion in the liver.

As with G-hesperidin, hesperidin and hesperetin also suppressed apo B secretion from HepG2 cells incubated in the presence of oleate (Fig. 4). Hesperetin, the aglycon of hesperidin and G-hesperidin, was markedly effective (Fig. 4). Chiba et al. have described the the detection of hesperetin in the serum of mice fed G-hesperidin (36). Moreover, it has been reported that G-hesperidin is hydrolyzed to hesperidin by brush border $\alpha$-glucosidases $(4,5)$ and that orally administered hesperidin is hydrolyzed to hesperetin by $\beta$-glucosidases from intestinal bacteria before absorption (Fig. 3) (1, 28-30). Taken together, these findings suggest that the suppressive effect of apo B secretion by G-hesperidin is due to hesperetin released through a sequence of enzymatic hydrolysis reactions. Since HepG2 cells have been known to possess $\alpha$-glucosidases and $\beta$-glucosidases $(37,38)$, it seems likely that these enzymes convert Ghesperidin to hesperetin in the cells. Thus, this cell model system is thought to resemble the physiological conditions in vivo, in terms of G-hesperidin availability.

For sufficient suppression of apo B secretion, G-hesperidin was required to be supplied to the cells during the oleate stimulation period (Fig. 5). Therefore, G-hesperidin is considered to act in the processes of cell responses caused by oleate stimulation and to result in the reduction of apo B secretion. This was further clarified by comparing the effects of G-hesperidin on apo B secretion from HepG2 cells incubated in the presence and absence of oleate. As shown in Fig. 6, we observed that G-hesperidin significantly suppressed apo B secretion only in the oleate-stimulated cells and failed to act on the cells incubated in the absence of oleate. This result indicates that G-hesperidin preferentially down-regulates the cell responses augmented by oleate stimula- 
tion. In the previous clinical study, G-hesperidin was effective in the hypertriglyceridemic subjects and significantly lowered their serum TG and apo B levels, but it had no effect on the normal subjects (11). Hypertriglyceridemic patients have been reported to experience an increase in VLDL secretion by excess influx of fatty acids, which consist mainly of oleic acid, into the liver (39). On this account, the present result that G-hesperidin preferentially reduces oleate-stimulated apo B secretion agrees closely with the observation in the previous clinical study (11).

It is not clear which process of the cell responses augmented with oleate is affected by G-hesperidin. However, we found that G-hesperidin significantly reduced the cellular CE amount in the oleate-stimulated HepG2 cells (Fig. 7). It has been known that oleate enhances the assembly of apo B-containing lipoproteins in HepG2 cells through the augmentation of TG and CE syntheses and results in increasing apo B secretion from the cells $(17,31,32)$. Furthermore, many investigators have shown that an increased apo B secretion is regulated by the cellular lipid syntheses. For instance, the reduction of TG or CE synthesis has been reported to prevent the assembly of apo B-containing lipoproteins, thereby decreasing apo B secretion from the cells $(17,31,32)$. On the basis of these findings, it is suggested that G-hesperidin down-regulates the assembly of apo B-containing lipoproteins via the reduction of CE synthesis augmented with oleate and that this effect contributes to the suppression of excess apo B secretion from the cells.

It is well known that acyl-CoA:cholesterol acyltransferase (ACAT) plays a critical role in CE synthesis (4O). Additionally, it has been demonstrated that ACAT activity in cultured hepatocytes is augmented by oleate stimulation and that this augmentation is attributed to the increase of mRNA level (41). Hence, there is a possibility that G-hesperidin exhibits its effect through the inhibition of ACAT activity augmented by oleate stimulation. In fact, it is known that several ACAT inhibitors prevent the assembly of apo B-containing lipoproteins in HepG2 cells by the reduction of CE synthesis and result in the suppression of apo B secretion $(17,42)$. Moreover, the supplementation of hesperidin has been shown to lower hepatic ACAT activity in hyperlipidemic rats (43). In addition, Borradaile et al. (44) and Wilcox et al. (45) have shown that hesperetin reduces apo B secretion from HepG 2 cells by inhibiting ACAT2 activity at the level of transcription. Based on these findings, gene analyses focused on ACAT are in progress in our laboratory.

As mentioned above, TG synthesis also participates in the regulation of apo B secretion and its inhibitors such as Triacsin D have been reported to suppress remarkably apo B secretion from HepG2 cells by decreasing the assembly of apo B-containing lipoproteins (32). However, it is unlikely that G-hesperidin suppresses apo B secretion through the inhibition of TG synthesis because this flavonoid derivative had no effect on cellular TG amount in the oleate-stimulated HepG2 cells (Fig. 7).
It is very important that G-hesperidin caused no change in cellular TG amount. Certain ACAT inhibitors have been reported to elicit an increase in cellular TG amount with the reduction of CE synthesis (17). This increase has been considered to be due to oleate, which is utilized for TG synthesis instead of a reduced CE synthesis and results in facilitating cellular TG accumulation (17). Nevertheless, it seems likely that G-hesperidin does not cause such augmentation of TG synthesis. Although the reason is unclear, it is possible that G-hesperidin leads to the enhancement of fatty acid $\beta$-oxidation as well as the reduction of $\mathrm{CE}$ synthesis. Flavonoid compounds such as catechins are well known to increase fatty acid $\beta$-oxidation in the liver (46). Therefore, we recently started investigations monitoring the changes in the mRNA expression of fatty acid $\beta$-oxidation enzymes.

It has been reported that apo B mRNA has a long half-life, which is approximately $16 \mathrm{~h}$, and that its level is not altered by various stimulations (47). From this finding, it has been considered that apo B synthesis is not transcriptionally controlled but is constitutively brought about in the hepatocytes (47). In addition, Wilcox et al. have shown that although hesperetin suppresses apo B secretion from HepG2 cells, it causes no change in apo B mRNA level (45). Hence, it is unlikely that G-hesperidin directly reduces apo B synthesis. On the other hand, the amount of synthesized apo B has been known to be modified by post-translational events; it has been reported that this apolipoprotein undergoes rapid intracellular degradation by the prevention of complexing with TG or CE $(22,33-35)$. We also observed that G-hesperidin treatment significantly decreased cellular apo B amounts in oleate-stimulated HepG2 cells (Fig. 8). This observation indicates that Ghesperidin prevents the assembly of apo B-containing lipoproteins by reducing $\mathrm{CE}$ synthesis and results in the augmentation of intracellular apo B degradation.

In conclusion, the present study has indicated that Ghesperidin down-regulates the assembly of apo B-containing lipoproteins via the reduction of cellular CE synthesis augmented with oleate and results in suppressing excess apo B secretion from HepG2 cells. This effect is speculated to be associated with the improvement of VLDL metabolic abnormality in hypertriglyceridemic subjects and considered as a mechanism of lowering serum TG level.

\section{Acknowledgments}

We are grateful to Ms. Michiyo Tsujita for her excellent technical and secretarial assistance in this study.

This work was supported in part by the Technical Development Program for developing agribusiness in the form of utilizing the concentrated know-how from the Private Sector from the Ministry of Agriculture, Forestry and Fisheries of Japan.

\section{REFERENCES}

1) Garg A, Garg S, Zaneveld LJD, Singla AK. 2001. Chemistry and pharmacology of the citrus bioflavonoid hes- 
peridin. Phytother Res 15: 655-669.

2) Galati EM, Trovato A, Kirjavainen S, Forestieri AM, Rossitto A, Monforte MT. 1996. Biological effects of hesperidin, a citrus flavonoid (Note III): Antihypertensive and diuretic activity in rat. IL Farmaco 51: 219-221.

3) Galati EM, Monforte MT, Kirjavainen S, Forestieri AM, Trovato A, Tripodo MM. 1994. Biological effects of hesperidin, a citrus flavonoid (Note I): Antiinflammatory and analgesic activity. IL Farmaco 49: 709-712.

4) Hijiya H, Miyake T. 1991. Alpha-glycosyl hesperidin, and its preparation and uses. European Patent Publication No. 0402049.

5) Kometani T, Terada Y, Nishimura T, Takii H, Okada S. 1994. Transglycosylation to hesperidin by cyclodextrin glucanotransferase from an alkalophilic Bacillus species in alkaline $\mathrm{pH}$ and properties of hesperidin glycosides. Biosci Biotechnol Biochem 58: 1990-1994.

6) Yamada M, Mitsuzumi H, Tsuzaki Y, Miwa Y, Chaen H, Yamamoto I. 2003. Antioxidant activity of glycosylated vitamin $\mathrm{P}$ and its suppressive effect on oxidative stress in hyperlipidemic mice. J Jpn Soc Nutr Food Sci 56: 355363.

7) Ohtsuki K, Abe A, Mitsuzumi H, Kondo M, Uemura K, Iwasaki Y, Kondo Y. 2002. Effects of long-term administration of hesperidin and glucosyl hesperidin to spontaneously hypertensive rats. J Nutr Sci Vitaminol 48: 420422.

8) Ohtsuki K, Abe A, Mitsuzumi H, Kondo M, Uemura K, Iwasaki Y, Kondo Y. 2003. Glucosyl hesperidin improves serum cholesterol composition and inhibits hypertrophy in vasculature. J Nutr Sci Vitaminol 49: 447-450.

9) Nishizaki Y, Tatefuji T, Akamatsu S, Hanaya T, Arai S, Ikeda M, Kurimoto M. 1997. Anti-allergic effect of $\alpha$ glucosyl-hesperidin. Med Biol 135: 199-204.

10) Miwa Y, Yamada M, Sunayama T, Mitsuzumi H, Tsuzaki Y, Chaen H, Mishima Y, Kibata M. 2004. Effects of glucosyl hesperidin on serum lipids in hyperlipidemic subjects: preferential reduction in elevated serum triglyceride level. J Nutr Sci Vitaminol 50: 211-218.

11) Miwa Y, Mitsuzumi H, Sunayama T, Yamada M, Okada K, Kubota M, Chaen H, Mishima Y, Kibata M. 2005. Glucosyl hesperidin lowers serum triglyceride level in hypertriglyceridemic subjects through the improvement of very low-density lipoprotein metabolic abnormality. $J$ Nutr Sci Vitaminol 51: 460-470.

12) Takata K. 2001. The metabolic regulation of TG-rich lipoproteins. Nippon Rinsho 59 (Suppl 2): 472-476.

13) Daubresse JC, Machowski R, Pulinx E. 1994. Efficacy of simvastatin for lowering cholesterol in non-insulin dependent diabetic patients with hypercholesterolemia. Acta Clin Belg 49: 68-75.

14) Sone H, Takahashi A, Shimano H, Ishibashi S, Yoshino G, Morisaki N, Saito Y, Kawazu S, Teramoto T, Fujita T, Shiba T, Iwamoto Y, Kuzuya N, Akanuma Y, Yamada N. 2002. HMG-CoA reductase inhibitor decreases small dense low-density lipoprotein and remnant-like particle cholesterol in patients with type-2 diabetes. Life Sci $\mathbf{7 1}$ : 2403-2412.

15) Olofsson SO, Asp L, Boren J. 1999. The assembly and secretion of apolipoprotein B-containing lipoproteins. Curr Opin Lipidol 10: 341-346.

16) Boren J, Rustaeus S, Olofsson SO. 1994. Studies on the assembly of apolipoprotein B-100- and B-48-containing very low density lipoproteins in McA-RH7777 cells. J Biol Chem 269: 25879-25888.
17) Wilcox LJ, Barrett PHR, Newton RS, Huff MW. 1999. ApoB 100 secretion from HepG2 cells is decreased by the ACAT inhibitor CI-1011: an effect associated with enhanced intracellular degradation of apoB. Arterioscler Thromb Vasc Biol 19: 939-949.

18) Haghpassand M, Wilder D, Moberly JB. 1996. Inhibition of apolipoprotein B and triglyceride secretion in human hepatoma cells (HepG2). J Lipid Res 37: 1468-1480.

19) Ellsworth JL, Erickson SK, Cooper AD. 1986. Very low and low density lipoprotein synthesis and secretion by the human hepatoma cell line Hep-G2: effects of free fatty acid. J Lipid Res 27: 858-874.

20) Thrift RN, Forte TM, Cahoon BE, Shore VG. 1986. Characterization of lipoproteins produced by the human liver cell line, HepG2, under defined conditions. J Lipid Res 27: 236-250.

21) Gibbons GF. 1994. A comparison of in-vitro models to study hepatic lipid and lipoprotein metabolism. Curr Opin Lipidol 5: 191-199.

22) Zhu D, Ganji SH, Kamanna VS, Kashyap ML. 2002. Effect of gemfibrozil on apolipoprotein B secretion and diacylglycerol acyltransferase activity in human hepatoblastoma (HepG2) cells. Atherosclerosis 164: 221228.

23) Arrol S, Mackness MI, Durrington PN. 2000. The effects of fatty acids on apolipoprotein B secretion by human hepatoma cells (HEP G2). Atherosclerosis 150: 255264.

24) Dixon JL, Furukawa S, Ginsberg HN. 1991. Oleate stimulates secretion of apolipoprotein B-containing lipoproteins from Hep G2 cells by inhibiting early intracellular degradation of apolipoprotein B. J Biol Chem 266: 5080-5086.

25) Bligh EG, Dyer WJ. 1959. A rapid method of total lipid extraction and purification. Can J Biochem Physiol 37: 911-917.

26) Carr TP, Andresen CJ, Rudel LL. 1993. Enzymatic determination of triglyceride, free cholesterol, and total cholesterol in tissue lipid extracts. Clin Biochem 26: 39-42.

27) Bradford MM. 1976. A rapid and sensitive method for the quantitation of microgram quantities of protein utilizing the principle of protein-dye binding. Anal Biochem 72: $248-254$.

28) Ameer B, Weintraub RA, Johnson JV, Yost RA, Rouseff RL. 1996. Flavanone absorption after naringin, hesperidin, and citrus administration. Clin Pharmacol Ther 60: 34-40.

29) Matsumoto H, Ikoma Y, Sugiura M, Yano M, Hasegawa Y. 2004. Identification and quantification of the conjugated metabolites derived from orally administered hesperidin in rat plasma. J Agric Food Chem 52: 66536659.

30) Manach C, Morand C, Gil-Izquierdo A, BouteloupDemange C, Rémésy C. 2003. Bioavailability in humans of the flavanones hesperidin and narirutin after the ingestion of two doses of orange juice. Eur J Clin Nutr 57: 235-242.

31) Cianflone KM, Yasruel Z, Rodriguez MA, Vas D, Sniderman AD. 1990. Regulation of apoB secretion from HepG2 cells: evidence for a critical role for cholesteryl ester synthesis in the response to a fatty acid challenge. J Lipid Res 31: 2045-2055.

32) Wu X, Sakata N, Lui E, Ginsberg HN. 1994. Evidence for a lack of regulation of the assembly and secretion of apolipoprotein B-containing lipoprotein from HepG2 
cells by cholesteryl ester. I Biol Chem 269: 1237512382.

33) Wilcox LJ, Barrett PHR, Huff MW. 1999. Differential regulation of apolipoprotein B secretion from HepG2 cells by two HMG-CoA reductase inhibitors, atorvastatin and simvastatin. J Lipid Res 40: 1078-1089.

34) Macri J, Adeli K. 1997. Conformational changes in apolipoprotein B modulate intracellular assembly and degradation of apoB-containing lipoprotein particles in HepG2 cells. Arterioscler Thromb Vasc Biol 17: 29822994.

35) Mohammadi A, Macri J, Newton R, Romain T, Dulay D, Adeli K. 1998. Effects of atorvastatin on the intracellular stability and secretion of apolipoprotein B in HepG2 cells. Arterioscler Thromb Vasc Biol 18: 783-793.

36) Chiba H, Uehara $M$, Wu J, Wang X, Masuyama R, Suzuki K, Kanazawa K, Ishimi Y. 2003. Hesperidin, a citrus flavonoid, inhibits bone loss and decreases serum and hepatic lipids in ovariectomized mice. J Nutr 133: 1892-1897.

37) Wisselaar HA, van Dongen JM, Reuser AJ. 1989. Effects of N-hydroxyethyl-1-deoxynojirimycin (BAY m 1099) on the activity of neutral- and acid alpha-glucosidases in human fibroblasts and HepG2 cells. Clin Chim Acta 182: 41-52.

38) Gebhardt R. 2002. Inhibition of cholesterol biosynthesis in HepG2 cells by artichoke extracts is reinforced by glucosidase pretreatment. Phytother Res 16: 368-372.

39) Shimakata T. 2001. Triacylglycerol, diacylglycerol, monoacylglycerol. Nippon Rinsho 59 (Suppl 2): 21-28.

40) Chang TY, Chang CC, Cheng D. 1997. Acyl-coenzyme A:cholesterol acyltransferase. Annu Rev Biochem 66: 613-638.
41) Seo T, Oelkers PM, Giattina MR, Worgall TS, Sturley SL, Deckelbaum RJ. 2001. Differential modulation of ACAT1 and ACAT2 transcription and activity by long chain free fatty acids in cultured cells. Biochemistry $\mathbf{4 0}$ : 4756-4762.

42) Musanti R, Giorgini L, Lovisolo PP, Pirillo A, Chiari A, Ghiselli G. 1996. Inhibition of acyl-CoA:cholesterol acyltransferase decreases apolipoprotein B-100-containing lipoprotein secretion from HepG2 cells. J Lipid Res 37: 1-14.

43) Bok SH, Lee SH, Park YB, Bae KH, Son KH, Jeong TS, Choi MS. 1999. Plasma and hepatic cholesterol and hepatic activities of 3-hydroxy-3-methyl-glutaryl-CoA reductase and acyl CoA:cholesterol transferase are lower in rats fed citrus peel extract or a mixture of citrus bioflavonoids. J Nutr 129: 1182-1185.

44) Borradaile NM, Carroll KK, Kurowska EM. 1999. Regulation of HepG2 cell apolipoprotein B metabolism by the citrus flavanones hesperetin and naringenin. Lipids 34: 591-598.

45) Wilcox LJ, Borradaile NM, de Dreu LE, Huff MW. 2001. Secretion of hepatocyte apoB is inhibited by the flavonoids, naringenin and hesperetin, via reduced activity and expression of ACAT2 and MTP. J Lipid Res 42: 725-734.

46) Murase T, Nagasawa A, Suzuki J, Hase T, Tokimitsu I. 2002. Beneficial effects of tea catechines on dietinduced obesity: stimulation of lipid catabolism in the liver. Int J Obes Relat Metab Disord 26: 1459-1464.

47) Hisatomi A, Yamamoto K. 2001. Apolipoprotein B (apoB100, apoB48). Nippon Rinsho 59 (Suppl 2): 109113. 